IRMA HERNANDEZ LÓPEZ

CIUDAD JUÁREZ, MÉXICO.

PSICOLOGA.CRIMINALISTA@GMAIL.COM

Fecha de recepción: 27/01/2021

Fecha de aceptación: 26/05/2021

Cómo citar: Hernández, I. (2021).

Especialista en diseño para retos sociales:

¿sujeto, actor y agencia/agente?

RChD: creación y pensamiento, 6(10), 1-15.

https//doi.org/10.5354/0719-837X.2021.63945

\section{Especialista en Diseño para retos sociales: ¿sujeto, actor y agencia/agente?}

Design specialist for social challenges:

Subject, Actor and Agency/Agent?

Resumen. En este artículo se reflexiona en torno al cuestionamiento: ¿es el diseñador/a sujeto, actor o agente en retos sociales? Una vez ubicada la posición que asume el diseñador/a en el reto de Diseño, se determinan sus decisiones e impacto de su propuesta diseñística. El rol en el que se desempeñe tendrá un impacto directo en el objetivo de las propuestas que plantea. Tomo como ejemplo el reto de la revictimización para analizar e ilustrar las diferencias al asumir un rol de sujeto, actor o agencia. Este estudio se hace mediante la revisión de la literatura y los resultados se presentan en una tabla comparativa. Se concluye que es primordial que el/la especialista en Diseño defina y reconozca, de inicio a fin, el rol que asumirá para abordar un reto de Diseño, el cual guiará su proceso diseñístico. Las decisiones de los diseñadores/as tienen un impacto directo en las personas y comunidades que puede ser positivo o negativo. En el caso de estudio, puede mitigar la revictimización o, por el contrario, acrecentarla.

Palabras clave: actor, agencia, retos sociales, revictimización, sujeto

\begin{abstract}
This article reflects on the question: Is the designer a subject, actor or agent in social challenges? Once designers define their role in the Design challenge, it determines their decisions and the impact of their Design solution. The role that the designer plays will have a direct impact on the goal of the potential proposal. The challenge of revictimization is taken as an example to analyze and illustrate the differences when assuming a role of subject, actor or agent. The analysis is based on a literature review and the results are introduced in a comparative table. It is concluded that it is essential that designers define and acknowledge, from start to finish, the role they will assume in the Design challenge because it will guide their Design process. Designer's decisions have a direct positive or negative impact on people and communities. In the case study example, it can mitigate revictimization or, on the contrary, increase it.
\end{abstract}

Keywords: actor, agency, revictimization, social challenges, subject 


\section{Introducción}

En los últimos años, México ha sido señalado como un país en donde se perpetran crímenes de lesa humanidad (Open Society Foundations, 2016) y se cometen serias violaciones a los derechos humanos (Amnistía internacional, 2018). Es un problema recurrente: los actos de revictimización tienen lugar en las instituciones, que son cometidos por el personal que tiene la encomienda de impartir justicia y atención. Estas revictimizaciones se manifiestan en el alto porcentaje de cifra negra ( $92,4 \%$, según el Instituto Nacional de Estadística, Geografía e Informática [INEGI], 2020). Las víctimas no denuncian por miedo a las represalias y a ser criminalizadas, o por la pérdida de tiempo, atención deficiente y largos tiempos de espera (INEGI, 2019). Además, las personas abandonan su proceso, entre otras cosas, por el mal trato que reciben en las instituciones (revictimización), o porque no sienten seguridad y confianza en las instalaciones donde son atendidas (Hernández, 2020).

Tradicionalmente, las disciplinas que se han enfocado en el estudio de la atención a víctimas y revictimización han sido principalmente el Derecho y la Medicina. En el ámbito institucional ha tomado relevancia la Psicología, Trabajo Social, y en los últimos años la Victimología. Se ha dejado de lado otras disciplinas que pueden contribuir de manera importante en la problemática de la revictimización, que se presenta no solo en México y América Latina, sino en el mundo entero.

En los últimos años, disciplinas como el Diseño y la Arquitectura han hecho importantes aportes para lidiar con problemáticas complejas como la revictimización, las víctimas y el Diseño de servicios en la atención a víctimas (Sanz-Abbud \& Saldarriaga, 2010; Hernández, 2011; Dorst et al., 2016; Minoja, 2016; Ortiz \& Hernández, 2019). Algunos de estos trabajos han propuesto el rediseño de los espacios como una medida para evitar la revictimización. Otras han puesto en la mesa de debate cuestionamientos en torno a la estética en la atención a víctimas (Hernández, 2019).

En este artículo se reflexiona en torno al rol de el/la diseñador/a como sujeto, actor o agente en el marco del reto de la revictimización. El artículo está estructurado en cinco secciones. En la primera, se expone el el marco teórico, se abordan los conceptos básicos: a) revictimización, que se define desde un enfoque victimológico; b) sujeto, c) actor y d) agencia. Por la misma tradición epistemológica de los términos, se abordan desde la disciplina de las Ciencias Sociales. Actor y agencia son conceptos que se han abordado desde la postura de la Ciencia Política, se considera que es una disciplina que ha contribuido de manera importante en el desarrollo de estos conceptos y para fines del artículo resulta ser un enfoque adecuado. En la segunda sección se expone brevemente la metodología. En la tercera se analizan trabajos desarrollados en torno a la temática de revictimización y víctimas asociadas al Diseño, se contrastan con la revisión de los planteamientos teóricos y se presenta una tabla con el resumen de los principales resultados y su análisis. En la sección cuatro y cinco, respectivamente, se presenta la discusión para cerrar con las conclusiones, donde se exponen los hallazgos y recomendaciones para investigaciones futuras. 


\section{Marco teórico}

\section{Revictimización y atención a víctimas}

La revictimización, también llamada victimización secundaria o doble victimización, se refiere a los costes personales que tiene para las víctimas la intervención del sistema de justicia (ministerio público, jueces, policías, etc.) y de instituciones como las agencias de investigación o instituciones de salud, que incrementan los padecimientos y sufrimientos de la víctima (Gutiérrez de Piñeras, Coronel \& Pérez, 2009). En la mayoría de los casos, las instituciones son agentes que criminalizan a las víctimas y no les proporcionan un trato apegado a los derechos humanos y victimales. Las víctimas se enfrentan a los altos niveles de burocratización, indiferencia, falta de tacto e incredulidad de algunos profesionales (Landrove, 1998, citado en Morillas, 2018). Esto provoca que la experiencia de las víctimas sea todavía más traumática para muchas de ellas (Marchiori, 2007). En síntesis, la revictimización es producto de una mala o inadecuada atención (Beristain, 1999). Esto se traduce en un abandono institucional, porque no se cumple con las funciones sustantivas encomendadas por el aparato legal (Sánchez, 2018). Este abandono también se refleja en el diseño y mantenimiento de las instalaciones, pasillos, iluminación, mobiliario, ubicación del inmueble, que son factores que potencializan la revictimización (Arrieta, 2014).

Algunos de estos factores revictimizantes como el Diseño del servicio o el Diseño de los espacios e infraestructura no son competencia directa de disciplinas que tradicionalmente se han dedicado a la atención de víctimas (Psicología, Derecho, Trabajo Social, Medicina). Sin embargo, la victimología y la Psicología han señalado el impacto negativo que tienen estos elementos en las víctimas. Es precisamente en este vacío donde el/la especialista en Diseño tiene importantes aportes por hacer.

Al ser la atención a víctimas un derecho fundamental y un aspecto esencial para su bienestar y protección (Melup, 2007), es prudente y necesaria una colaboración entre las disciplinas para enfrentar el reto de la revictimización y poner en el centro a las propias víctimas para establecer un acercamiento y una atención con dignidad, enfocada a una comunicación clara, honesta, sin minimizar su dolor (Beristain, Valencia, Buitrago \& Cox, 2017). Esto tendría que estar reflejado en cómo (Diseño de servicios) y dónde (instalaciones) son atendidas las personas, de tal forma que se puedan reducir o eliminar los factores que llevan a la revictimización.

Estos factores revictimizantes demandan la atención de otras disciplinas como la Arquitectura y el Diseño. Es necesario explorar el rol que las/los especialistas de estas disciplinas asumen dentro de la problemática de la revictimización. En este artículo nos enfocamos a los y las diseñadores/as. Los tres roles que se exploran en los apartados posteriores son: a) el especialista como sujeto, b) el especialista como actor, y c) el especialista como agente/agencia.

\section{Sujeto}

En el sujeto se deposita la acción, e implica dos escenarios relevantes: que tenga o no derechos (Ema, 2004), en función de cómo es percibido en determinados repertorios discursivos, contextos temporales y geográficos. 
Este autor destaca dos posturas: el sujeto como territorio y consecuencia de la propia acción, y el sujeto que presenta la "noción de agencia como privilegio de conectarse y moverse (verbos) frente a las (id)entidades (nombres), para explicar la acción" (Ema, 2004, p.3). En la primera, el sujeto es depósito de algo. En la segunda postura, el sujeto es visto como ente activo con limitantes.

[El sujeto es...] una entidad sujetada al discurso y las instituciones (en el primer Foucault) o a la ideología (Althusser) o a la estructura lingüística (Levi-Strauss). El sujeto es una fuente de acción. La capacidad de acción del sujeto no es otra cosa que la posibilidad de poder actuar modificando la regla que le precede y le constituye. (Ema, 2004, p.10-11)

En esta misma línea se ubica la postura de Arend (citado en Botero \& Leal, 2015), que hace referencia al sujeto político y lo describe como un ser libre, único e impredecible; la acción y el discurso son dos factores que lo definen. Tiene una libertad limitada.

\section{El/la diseñador/a como sujeto}

Con base en las propuestas teóricas anteriores, se entiende que el especialista en Diseño en un rol de sujeto, es depositario de la acción y está sujeto a las demandas y necesidades de la empresa, empleador, institución. También el/la diseñador/a tiene una capacidad de acción limitada, es visto como un interlocutor. Para adoptar tal rol “... es importante saber quién es nuestro interlocutor y qué expectativas se tienen frente al discurso [reto de Diseño]. También, juega un papel importante el grado de empatía que exista entre los interlocutores: cuanta más confianza y conocimiento, menos distancia comunicativa [mayor comprensión del reto y de la integración de ideas de los diferentes especialistas]" (Fajardo, 2009, p. 1245).

"Interlocutores: son los participantes directos en el acto de la comunicación y están marcados por la oposición entre la primera y la segunda persona del singular (yo/tú). Pueden denominarse de diversas formas: enunciador/enunciatario, emisor/receptor, locutor/a locutario o interlocutor, hablante/oyente, destinador/destinatario, etc." (Lastra, 2011 p. 169).

En este sentido, el/la diseñador/a es el destinatario del mensaje, de lo que el cliente, la empresa o la institución requiere. El sujeto es fuente de la acción, porque él es quien plasma las ideas y antecedente de la acción. Es el intermediario que hace posible que se materialice el objeto, servicio, artefacto, etcétera. Si bien puede tomar algunas decisiones de Diseño, éstas están apegadas a la solicitud explícita de quien le depositó la tarea. La libertad del especialista se encuentra limitada y restringida a los intereses, necesidades, caprichos o demandas del depositario. Los límites de la autonomía del sujeto están dados a partir de lo acordado y establecido, durante la interlocución que mantuvo con la empresa o empleador que lo contrató.

Actor

El concepto de actor es ampliamente utilizado en las ciencias sociales así como en los discursos de políticos y periodistas (García, 2007). La acepción 
del diccionario de la Real Academia Española (2020) señala que actor es aquel que actúa. Dentro de ese actuar, la literatura destaca factores como autonomía, poder, libertad. Habría que preguntarse ¿cómo ejerce el diseñador/a su autonomía, poder y libertad para enfrentar los retos de Diseño? ¿Ellos escogen los retos, les son asignados? En este sentido, Crozier (1990) hace énfasis en que la actuación y autonomía del actor depende del contexto y de la posición que ocupa. Los actores dependen unos de otros para el cumplimiento de los objetivos, y esto se da mediante un actuar comprometido entre ellos, la negociación, el intercambio de ideas, posiciones y propuestas. El actor no es estático. Cambia de posición, es flexible y está abierto a la retroalimentación y argumentación. Todo actor tiene poder y, lejos de la imposición, esto es visto como un principio para una actuación organizada y dirigida del equipo de trabajo, para el cumplimiento de los objetivos de Diseño. El poder que se ejerce entre los actores, en lo individual o en lo colectivo, tiene la posibilidad de actuar sobre otros individuos o un grupo. El/la diseñador/a como actor es capaz de influir a los otros. Es importante reiterar que el poder del actor diseñador/a es ejercido independientemente de la posición jerárquica en que se encuentre, en tanto "todo actor es relativamente autónomo" (Crozier, 1990, p.27), porque cuenta con estrategias para afrontar las situaciones que se le presenten. Estas estrategias están sustentadas por la experiencia, conocimientos, racionalidad y habilidades que pueden o no contribuir a resolver las situaciones de la mejor manera.

\section{El/la diseñador/a como actor}

El/la diseñador/a en su rol de actor responde a necesidades de un entorno, de una persona, o una institución, sin despojarse totalmente de sus propios valores, normas y creencias.

García (2007) identificó que las decisiones de los actores dependen de: a) la posición formal que ocupa el actor, es decir el cargo, la responsabilidad o el puesto que desempeña; b) sus intereses, implica reconocer qué lo lleva a actuar de una forma u otra, porque adoptar una perspectiva para llevar a cabo el proceso diseñístico, tiene que ver con las expectativas; c) sistema de creencias y valores normativos que pueden restringir "las opciones que los decisores barajan como aceptables y legítimas desde el punto de vista moral" (García, 2007, p. 11); d) habilidades, tiene que ver con la formación académica, experiencia profesional, incluso personalidad para tener o no éxito en la toma de decisiones y la puesta en práctica. "Las habilidades no se refieren sólo a la competencia para resolver problemas o para planificar actividades de manera estratégica, sino también a la aptitud que se demuestre en las relaciones con otros actores o a la destreza para hacer del entorno un escenario favorable a los intereses propios, aprovechando las oportunidades que le brinde y salvando las restricciones y obstáculos que le ofrezca" (García, 2007, p. 12); e) las oportunidades que le brinda el entorno para poder actuar, en este punto se pueden señalar los apoyos (entre colegas, presupuestario, trabajo colaborativo, etc.).

En la medida en que el/la diseñador/a tenga un cargo de liderazgo en el proyecto podrá tomar las decisiones. Se espera que entre mayor sea el puesto de responsabilidad, mayor margen de maniobra tenga el actordiseñador/a para tomar decisiones (García, 2007). 
En el actor se conjuga todo un sistema de creencias que puede potencializar las soluciones de Diseño u obstaculizarlas. Su sistema de creencias interviene en cómo asume el reto diseñístico; definir qué postura de Diseño adopta para las propuestas de solución; establecer qué tiene valor, relevancia, por dónde empezar, hasta dónde intervenir y hasta donde se tiene injerencia. El actor pone sus propios límites y alcances. El actor no se despoja de su ser, de su persona ni de su subjetividad, y está reflejado en cómo asume el reto y cómo lo resuelve; tanto en el proceso de Diseño como en el resultado final.

\section{Agencia/agente}

La Real Academia Española (RAE, 2020), señala que la agencia es la capacidad de los individuos para actuar de forma independiente y hacer sus elecciones de modo libre. Leiva (2015), menciona que el agente es quien participa de forma activa en los objetivos y metas que se proponen. Dentro de la agencia también hay otras personas, grupos e instituciones que intervienen y son parte de la ejecución de los procesos, por lo que contribuyen y favorecen en el cumplimiento de las metas. Ser agente implica, además de cumplir los objetivos personales/profesionales, cumplir objetivos sociales. "Una persona ejerce su aspecto de agencia cuando decide por sí misma, cuando actúa con autonomía. Las razones de las decisiones nacen de un proceso de deliberación, no se basan en meros caprichos o impulsos" (Leiva, 2015, p. 15).

Giddens (1984) señala con respecto a la agencia:

La agencia no se refiere a la intención que tiene la gente al hacer las cosas, sino a su capacidad de hacer esas cosas en primer lugar (por eso la agencia implica poder ... de un agente, como "alguien que ejerce poder o produce un efecto"). La agencia se refiere a eventos de los que un individuo es el perpetrador, en el sentido de que el individuo podría, en cualquier fase de una determinada secuencia de conducta, haber actuado de manera diferente. Pase lo que pase, no habría sucedido si ese individuo no hubiera intervenido. (p. 9, traducción de la autora).

Desde el enfoque de Ema (2004), la agencia es entendida como "entidades mediadoras que facilitan el ejercicio de determinadas acciones" (p. 17), por lo que agente es la persona mediadora pero con un alto grado de autonomía, sin caer en el autoritarismo. Se entiende que este actuar no es de una sola persona, no es producto individual, sino un trabajo conjunto, colaborativo, compartido. La capacidad mediadora de la agencia es lo que permite que haya una comunicación, interconexión de acontecimientos, diálogo, intercambio de ideas, prácticas para que se concreten en actos (Ema, 2004).

En síntesis, se destaca que la agencia/agente tiene la capacidad de actuar por sí mismo, tomar sus propias decisiones, apoyarse en los otros para el cumplimiento de metas profesionales y personales. Tiene incidencia en los otros (lo social), actúa con autonomía y es parte esencial de un todo. La agencia es compartida. 


\section{El/la diseñador/a como agente}

Para considerar al Diseño y el/la diseñador/a como un agente o una agencia se tiene que valorar la capacidad (conocimiento, habilidades, formación, interés, conciencia social) que tiene para enfrentar retos sociales, como la victimización. Esta capacidad tiene que estar dada desde la formación que reciben como especialistas en Diseño, y considerar los valores e intereses propios. Desde la visión de Giddens (1984), el ser agente/agencia conlleva la capacidad de ser parte activa en todo el proceso diseñístico; en todo momento tiene capacidad de decisión e influencia. Ser agente implica estar presente en todo el proceso que conlleva el Diseño de un producto, servicio o artefacto. El diseñador/a como agente participa en la definición de la tarea de Diseño, en vez de solo resolver una tarea específica encomendada por otros. Tiene la capacidad de cambiar las cosas. Su presencia, participación y aporte hacen una diferencia en la solución al reto planteado, y no se llegaría a ella sin su participación.

Desde la perspectiva de Ema (2004) se entendería que el Diseño, diseñador/a como agencia/agente, en su rol de mediador, tiene una responsabilidad sobre la acción, sobre la propuesta de Diseño. La responsabilidad que asume no es casualidad, sino un reconocimiento a su rol como un elemento movilizador de la transición, de la potencia al acto concreto. Tanto la disciplina del Diseño como el/la diseñador/a tienen una responsabilidad directa en el proceso de Diseño, porque las decisiones que toman impactarán en el proceso y resultado final. Así, la toma de decisiones debe tener un sustento y una razón de ser. El/la diseñador/a tiene además una responsabilidad directa, al dirigir el proceso y asumir un liderazgo que es avalado por su conocimiento especializado y modo de abordar el reto social. El/la diseñador/a actúa como responsable de un acto concreto, dentro del contexto de delimitación de determinadas condiciones de posibilidad. El/la diseñador/a no actúa solo/a, sino como parte de un todo. La agencia es compartida, es producto de la retroalimentación y el intercambio de ideas que dan paso a una solución integral, se da paso al trabajo desde un enfoque transdisciplinario.

En síntesis, cuando los diseñadores/diseñadoras asumen un rol de agencia/ agente asumen el reto de Diseño desde una perspectiva de "un quién o un qué-, a un proceso -a un cómo" (Ema, 2004, p.18).

En la Tabla I se sintetizan los tres conceptos revisados: sujeto, actor y agencia/agente.

En síntesis, se puede señalar que el diseñador como sujeto tiene una capacidad limitada de influir en la toma de decisiones para resolver el reto de Diseño. Es depositario de la tarea. Su capacidad de interlocución activa se ve muy limitada y está supeditada a los requerimientos del cliente, quien no necesariamente toma en cuenta el contexto en sus diferentes vertientes. En este rol, el diseñador/a debe ser empático y con una fuerte capacidad de escucha para traducir y plasmar las necesidades de su depositario en una solución diseñísitca (objeto, artefacto) que lo satisfaga.

A diferencia del sujeto, el/la diseñador/a actor tiene autonomía, poder y libertad, limitadas, pero con mayor capacidad de injerencia en otros. El poder 


\begin{tabular}{|c|c|c|c|c|c|c|c|}
\hline Término & Concepto & Autonomía/libertad & $\begin{array}{l}\text { Ejercicio } \\
\text { del poder }\end{array}$ & Contexto & $\begin{array}{l}\text { Relación Jerar- } \\
\text { quía }\end{array}$ & Interacción & $\begin{array}{l}\text { Caractrísticas del } \\
\text { diseñdor/a }\end{array}$ \\
\hline Sujeto & $\begin{array}{l}\text { Humano transparente, } \\
\text { autónomo y racional, } \\
\text { origen y fuente del } \\
\text { conocimiento (Ema, } \\
2004, \text { p. 2).) }\end{array}$ & Interlocutor activo & Limitado & Sí & Jerarquía & $\begin{array}{l}\text { Depositario } \\
\text { Activa limitada }\end{array}$ & $\begin{array}{l}\text { Empatía } \\
\text { Escucha }\end{array}$ \\
\hline Actor & $\begin{array}{l}\text { Tiene una actuación } \\
\text { estratégica y actúa } \\
\text { bajo sus orientaciones } \\
\text { (percepciones y } \\
\text { preferencias) y por sus } \\
\text { capacidades (Scharpf, } \\
\text { 1997, en García, 2007). } \\
\text { Tiene una intención y } \\
\text { un propósito (Mayntz y } \\
\text { Scharps, 1995, en García, } \\
\text { 2007). }\end{array}$ & $\begin{array}{l}\text { No totalmente, } \\
\text { relativa. }\end{array}$ & Sí & Sí & $\begin{array}{l}\text { Importante } \\
\text { para influir } \\
\text { en la toma de } \\
\text { decisiones. }\end{array}$ & $\begin{array}{l}\text { Primordial, el trabajo y } \\
\text { relación entre actores } \\
\text { (Valenzuela, 2012). }\end{array}$ & $\begin{array}{l}\text { Liderazgo } \\
\text { Toma de } \\
\text { decisiones } \\
\text { Integrar un equipo } \\
\text { de trabajo. }\end{array}$ \\
\hline $\begin{array}{l}\text { Agencia } \\
\text { / Agente }\end{array}$ & $\begin{array}{l}\text { Capacidad para hacer las } \\
\text { cosas. Es un proceso. }\end{array}$ & $\begin{array}{l}\text { Sí la tiene y trabaja en } \\
\text { conjunto con otras } \\
\text { instancias para la } \\
\text { toma de decisiones. }\end{array}$ & Sí & Sí & $\begin{array}{l}\text { Hay una relación } \\
\text { de iguales y } \\
\text { cada disciplina, } \\
\text { instancia } \\
\text { aporta desde su } \\
\text { expertise }\end{array}$ & $\begin{array}{l}\text { Continua entre } \\
\text { agentes y agencia. }\end{array}$ & $\begin{array}{l}\text { Formación, } \\
\text { capacidades } \\
\text { Mediador, } \\
\text { Concreción de } \\
\text { ideas. }\end{array}$ \\
\hline
\end{tabular}

Tabla 1. Roles que asume el/la diseñador/a (elaboración propia a partir del análisis de la literatura revisada).

Nota. Síntesis de los componentes que se identifican en los roles que ha asumido el/la especialista en Diseño en retos sociales, como la victimización. es utilizado para organizar y sacar a flote los objetivos diseñísticos. Se establece una relación más horizontal entre actores, hay un intercambio de ideas. Se toma en cuenta su experiencia, conocimientos y habilidades, lo cual le da legitimidad, y se establece un diálogo entre sus iguales y quienes lo contratan (empresa, institución). El contexto es otro factor que se toma en cuenta en el proceso de Diseño e influye en sus decisiones, que no están libres de sus valores e ideas.

Finalmente, el/la diseñador/a como agente asume una responsabilidad social, donde sus valores, ideales, posturas, conocimiento, habilidades, formación, interés y conciencia social son parte medular de sus intervenciones diseñísticas. Generalmente es el propio diseñador/a quien escoge, busca y selecciona los proyectos en los cuales quiere participar: propone, busca y realiza. El agente busca cumplir sus objetivos profesionales, personales y sociales, dentro de un trabajo colaborativo donde comparte la agencia. El compromiso de asumir un reto de Diseño va más allá de solo cumplir los objetivos; lo que busca es impactar en las personas y generar un cambio. Su objetivo es influir en los otros por medio de sus Diseños. Estos Diseños son producto de un análisis y toma de decisiones que tienen como referencia el contexto y es parte de una solución integral del reto asumido.

\section{Metodología}

Una vez revisada la literatura sobre sujeto, actor y agente/agencia, se analizaron y sintetizaron las ideas, orientándolas a la disciplina del Diseño y a sus especialistas. En el siguiente apartado se hace un análisis de trabajos de investigación empírica y teórica que han abordado el tema de la victimización desde el enfoque de Diseño. El objetivo es identificar el rol que han asumido las/los diseñadores para atender este reto social.

\section{Resultados $\mathrm{y}$ análisis}

En la Tabla 2, se presentan en orden cronológico, siete trabajos seleccionados bajo dos criterios: el primero, es que tengan relación con la disciplina del Diseño; 
Título de la

investigación

El Diseño estratégico Sanz y Saldarriaga

Autor

Rol Bueno (2010)

Agente

Interlocutor entre la problemática

y la organización.

Investigación previa para

determinar la viabilidad de

proyecto.

Integraron un equipo de trabajo

multidisciplinario.

Eje rector del trabajo fue una

metodología del Diseño.

Impacto directo a los usuarios

afectados y a la sociedad en

general.

Vincula sociedad, gobierno.

Centros de Justicia para

Hernández (2011)

Actor

Aún cuando el diseñador/a está

las Mujeres. Lineamien-

tos urbanos y diseño

arquitectónico

involucrado en todo el proceso, se

le visualiza como un medio para

lograr los fines. No se visualiza un proceso de intercambio de

ideas o retroalimentación. Da la

impresión de que es visto como

alguien que ejecuta las ideas de

otros. Se trabaja desde un enfoque

multidisciplinario, pero no hay una

interacción e intercambio de ideas.

Por otro lado, no es claro si se toma

en cuenta la opinión de las víctimas.

Diversos escenarios

Arrieta (2014)

Actor

Es parte activa del proceso,

judiciales y su impacto

en la victimización

secundaria

To Protect and Serve

Dorst et al. (2016)

Agencia/

agente

toma algunas decisiones con

base en el intercambio de ideas

y la normatividad que compete

al Diseño urbanístico y la

normatividad jurídica en el acceso

a la justicia y atención a víctimas.

Analizaron la problemática en su dimensión holística. Integraron un grupo de especialistas. Generaron una propuesta. Fueron parte de todo el proceso. Rol activo y toma de decisiones.

\begin{tabular}{|c|c|c|c|}
\hline $\begin{array}{l}\text { La inserción de la } \\
\text { disciplina en Diseño } \\
\text { en el reto de la } \\
\text { victimización }\end{array}$ & $\begin{array}{l}\text { Ortiz y Hernández } \\
(2019)\end{array}$ & Agencia & $\begin{array}{l}\text { Hace énfasis en la formación del } \\
\text { diseñador/a en problemas sociales } \\
\text { para el trabajo transdisciplinario. }\end{array}$ \\
\hline $\begin{array}{l}\text { La atención estética: } \\
\text { explorando las } \\
\text { posibilidades de } \\
\text { interacción para reducir } \\
\text { la revictimización }\end{array}$ & Hernández (2019) & Agencia & $\begin{array}{l}\text { Trabajo teórico que sitúa al } \\
\text { diseñador/a como una pieza } \\
\text { fundamental para la atención de } \\
\text { víctimas. Lo potencializa } \\
\text { como un agente de cambio. }\end{array}$ \\
\hline $\begin{array}{l}\text { Human-centric Design } \\
\text { Solutions to Prevent } \\
\text { Violence against } \\
\text { Women and Girls }\end{array}$ & $\begin{array}{l}\text { World Design } \\
\text { Organization (2020) }\end{array}$ & Actor & Mediadores. Rol activo. \\
\hline
\end{tabular}

el segundo, que los trabajos hayan abordado el tema de la revictimización y atención a víctimas. Los siete casos seleccionados cumplen con ambos criterios y permiten realizar el siguiente ejercicio: categorizar los trabajos en base a los conceptos definidos de agencia, sujeto y actor.

La Tabla 2 identifica en qué categoría se ubica la participación del Diseño en los trabajos analizados y visualiza cuáles fueron las características de esa participación.
Tabla 2. Clasificación del rol que asume el diseñador en el reto de la victimización y revictimización (elaboración propia, resultado del análisis de las investigaciones revisadas).

Nota. Se muestran los resultados del análisis de las investigaciones de propuestas de Diseño que hacen frente a la victimización y revictimización. 
1. Es importante reconocer que existe una larga tradición en la disciplina del Diseño, desde sus inicios y durante su desarrollo hasta los años noventa, como generadora de cambios e injerencia en las problemáticas sociales. Por ejemplo, la escuela Bauhaus hasta años recientes se enfoca en la victimización. La propuesta de aplicar el Diseño en los casos de atención a las víctimas para minimizar o anular la revictimización, es una muestra más de la potencialidad que ha tenido el Diseño como agente.
De los trabajos analizados, en ninguno se ubicó a los especialistas en Diseño como sujeto, lo cual es un importante hallazgo. Los resultados sugieren que el/la diseñador/a como sujeto no tiene cabida, por lo menos en esta problemática social, por la naturaleza misma del reto. El/la diseñador/a como sujeto está presente en la medida en que es condición indispensable tanto en el actor como en el agente para el distanciamiento cognitivo, de decisión y de proceso frente al reto abordado. Las problemáticas sociales requieren una postura activa, propositiva, holística, integradora de ideas entre los diferentes sectores involucrados, y también de disciplinas. La relación que establece el Diseño o diseñador/a con el resto de los involucrados es una relación dialéctica, bidireccional. Es diferente de la relación que se puede establecer con la iniciativa privada, que se caracteriza por una relación unidireccional, donde el cliente o empresa solicita al sujeto (diseñador) lo que requiere y éste ejecuta, dejando de lado la retroalimentación e intercambio de ideas. El especialista en Diseño es visto como sujeto de la acción: la persona que ejecutará un producto, servicio, artefacto para el cual fue contratado. Este diseño será acorde a los intereses de alguien más y, con base en el análisis previo, no considera los intereses del diseñador/a. Desde el enfoque de Ema (2004), el/la diseñador/a asume una postura de sujeto de acción. Su actuar está sujeto a un discurso, tal como lo menciona Foucault (en Ema, 2004), un discurso que está dado por la empresa. El/la diseñador/a participa con poca autonomía y retroalimenta las ideas que otros establecieron. Toma decisiones dentro de un margen restringido, cuida no salirse del pedido, solicitud o encargo para el cual fue contratado. Aun cuando tiene un rol de interlocutor, es limitada su participación. Es visto como el ejecutor de las ideas, el sujeto que es capaz de plasmar ideas en un objeto concreto. Su interlocución e intercambio de ideas se circunscribe a un otro que marca la directriz, y que no necesariamente toma en cuenta el contexto de tiempo, modo y lugar que se requiere para enfrentar retos sociales.

En la Tabla 2 se observa que, en la mitad de los proyectos, las/los investigadores/ as han proyectado tanto a la disciplina del Diseño como al diseñador/a como agentes y la otra como actores. Esto tiene importantes repercusiones positivas en cómo asumen los retos de Diseño y cómo los enfrentan.

- Se empieza, con mayor fuerza', a visualizar al Diseño como un factor de cambio e injerencia en problemáticas sociales como la revictimización.

- Se trabaja con un enfoque multidisciplinario.

- Los diseñadores/as actúan ante las problemáticas y retos sociales que demandan los tiempos actuales.

- Asume el poder, la libertad, la iniciativa y la autonomía para proponer temas de estudio que requieren soluciones integrales, sin dejar de lado el contexto en que tienen lugar (Crozier, 1990). Este contexto está dado en tiempo (cuándo), modo (cómo) y lugar (dónde). El contexto también ha sido considerado en términos de escalabilidad, a partir de cuatro sistemas relacionados: micro, meso, exo y macro (Bronfenbrenner, 1987). El contexto es complejo, abarca realidades y éstas son difícilmente aislables (Velasco Maillo \& Díaz de Rada, 1997). No se pueden ni deben ignorar.

Algunas propuestas de Diseño han sugerido el modelo ecológico para considerar cuatro dimensiones del contexto como áreas de oportunidad 
que facilitan asumir el rol de agente de cambio (Ortiz, Hernández \& Peinado, 2019). Dentro de este rol también se encuentran inmersos los valores, creencias, ideales y tal vez las posturas políticas de las y los especialistas en Diseño. Ser agente muestra la responsabilidad social individual y profesional. Las soluciones y las propuestas, además, son resultado del análisis, de un método, de un procedimiento y no por causa fortuita, o por capricho u ocurrencias, como lo menciona Leiva (2015). En este punto, el/ la diseñador/a supera el diseñar por diseñar. Los trabajos de Manzini (2015), Ortiz (2016) y Molina y Sánchez (2019), adquieren importante relevancia. El primero destaca diferentes roles de los especialistas en Diseño, de la práctica a la gestión. El segundo autor presenta algunas características que deben desarrollar los/las diseñadores/as para abordar retos sociales. Y por último Molina y Sánchez (2019) empiezan a plantear la idea de posicionar al diseñador/a como agente de cambio.

Otro factor relevante es que la disciplina del Diseño y sus especialistas, se vincula ${ }^{2}$ con organizaciones de la sociedad civil, instituciones de gobierno y el sector privado (empresas), y adquiere una mirada holística. Se asumen como agentes, pues además de satisfacer los objetivos y metas profesionales y personales, sus trabajos, proyectos y propuestas tienen un impacto social, o por lo menos se encaminan hacia ese objetivo. Se plantea que sea el/la diseñador/a quien busque los espacios y proyectos para incidir en ámbitos poco explorados, y que no espere a ser convocado/a por empresas, el gobierno y organizaciones de la sociedad civil.

\section{Discusión}

Con estos resultados no se pueden determinar totalmente los alcances del rol transformador del Diseño en problemáticas como la revictimización. Lo que sí se puede señalar con base en los resultados obtenidos es que existen diferencias relevantes en los tres roles analizados: sujeto, actor y agente. Considerar estas diferencias es una importante guía para dirigir las propuestas de Diseño hacia los resultados que se quieren lograr y, así delimitar el impacto y alcance que se quiere tener con las propuestas de Diseño. Ya sea desde un rol de sujeto, actor o agente, la disciplina del Diseño contribuye a fortalecer los derechos de las víctimas, e impacta en su bienestar, protección y trato digno (Melup, 2007; Beristain, Valencia, Buitrago \& Cox, 2017). No se duda del rol transformador que puede tener el Diseño, sin embargo, no todos los/las diseñadores/as y/o proyectos lo tienen automáticamente, pues está fuertemente relacionado con el tipo de tarea a realizar (desde la asignada hasta la definida por el/la diseñador/a). El mayor impacto se obtiene en el rol de agencia, pues las acciones consideran la problemática en su dimensión holística, integran a un grupo de especialistas, establecen el área de oportunidad y lo desarrollan. Forman parte de todo el proceso y tienen un rol activo y la posibilidad de tomar decisiones importantes para el proyecto en cuestión. Con un diseño metodológico apropiado, se podría determinar e incluso evaluar el impacto de las propuestas de Diseño, siempre que este sea el objetivo de el/la diseñador/a.

Es recomendable que la disciplina de Diseño haga un esfuerzo por abordar retos sociales como la revictimización y, que éstos se visualicen como oportunidades: abordarlos brinda a la disciplina y a las/los especialistas en
2. Si bien se tiene registro que los urbanistas, desde hace algunas décadas, ya habían establecido contacto con instituciones gubernamentales, administrativas y empresas, no se puede negar que éstas han tenido un tinte político. $Y$ tal vez sea el momento de que el diseño y sus especialistas asuman una postura concreta para fortalecer su rol como agentes. 
Diseño la oportunidad para integrarse a equipos transdisciplinarios en su aproximación. En estas condiciones, el Diseño integra sus saberes y creatividad $y$, así incide de manera positiva en la problemática para las víctimas, la sociedad y las instituciones. La literatura previa ha sugerido la relevancia de establecer condiciones idóneas para que los diseñadores/as definan un reto de Diseño; un punto de inicio es abordar un reto complejo (Ortiz, Hernández \& Peinado, 2019). Adoptar como marco de referencia el Diseño estratégico basado en soluciones, puede ser un medio para identificar e implementar nuevas oportunidades para minimizar y eliminar la revictimización, un fenómeno muy recurrente en las instituciones gubernamentales.

Este artículo propone que los/as diseñadores/as dialoguen, reflexionen o asuman un rol definido en una tarea de diseño, ya sea como sujeto (Ema, 2004), actor (Crozier, 1990; García, 2007) o agente (Ema, 2004; Leiva, 2015) y pongan en práctica los principios que implica su rol al momento de diseñar productos, herramientas, sistemas o servicios accesibles, funcionales y empoderadores, que contrarresten actos, conductas y circunstancias involucradas en el fenómeno de la revictimización. Además, propone que el diseñador/a reflexione y asuma diferentes roles (sujeto, actor y agencia) en proyectos de diseño que, potencialmente, le permitirán desarrollar habilidades para cada rol.

Tal como lo menciona Srinivasan (World Design Organization, 2020), se necesita reconocer que el diseño es una parte importante e integral del proceso de desarrollo. Se requiere gestionar ante el gobierno, la comunidad, los propios diseñadores/as, organizaciones de la sociedad civil y la comunidad científica, entre otros, que el Diseño se piense como un medio para resolver problemas. Este planteamiento es una invitación a que los especialistas en Diseño, y la propia disciplina, se conceptualicen en un rol de actores y agentes de cambio y transformación, más que meros sujetos e interlocutores pasivos en el proceso y soluciones de diseño.

\section{Conclusiones}

Se reconoce la complejidad del comportamiento humano. No se puede ser simplista al identificar el rol del diseñador/a en retos sociales complejos. Es relevante identificar y hacer énfasis en los tres roles discutidos en este artículo.

Este trabajo es un referente para continuar la exploración, exposición, ejemplificación y análisis del rol del diseñador/a para enfrentar retos sociales desde su ámbito profesional.

No es que el especialista tenga que asumir un rol en específico. La importancia está en reconocer su rol, para tener conciencia de las implicaciones que tiene su intervención en las soluciones que se plantean. Podrá saber de qué forma contribuye y el impacto que tendrá su participación. Si quiere continuar en ese mismo rol lo decidirá porque son los objetivos que persigue. Un rol no es mejor que otro, pero existen diferencias en el desempeño e impacto que tendrá un objeto, artefacto, producto, experiencia o servicio diseñado. El rol asumido sí influye en hasta dónde y cómo puede incidir el Diseño/diseñador/a en los retos sociales y por tanto impactar en las personas. 
Se hace énfasis en la importancia de realizar un trabajo transdisciplinario, de ahí que sea recomendable poner el esfuerzo en continuar con estas reflexiones y cuestionamientos desde las aulas hasta la práctica profesional. “... Tenemos que escudriñar nuevos terrenos para encontrar inspiración y cultivar el hábito de buscar personas, lugares, [situaciones], organizaciones, proyectos e ideas que no aparecen en el radar utilizado por nuestros capitanes allá arriba en el puente de mando" (Thackara, 2013, p. 168).

El/la diseñador/a tiene tres roles: sujeto, actor y agente. Asumirse como uno $\mathrm{u}$ otro tiene importantes diferencias y efectos, por lo que es indispensable reflexionar hacia dónde se tiene que dirigir la disciplina del Diseño, qué se espera de las/los diseñadores y cuál es el rol que los tiempos actuales demandan a la disciplina y a sus especialistas. 


\section{Referencias}

Amnistía Internacional. (2018). Informe 2017/2018. La situación de los derechos humanos en el mundo. https://www.amnesty.org/download/Documents/ POL1067002018SPANISH.PDF

Arrieta, V. (2014). Diversos escenarios judiciales y su impacto en la victimización secundaria. Eguzkilore, 28, 287-320.

Beristain, A. (1999). Criminología y Victimología. Leyer. Beristain, M., Valencia, A., Buitrago, A. \& Cox, A. (2017). Metodología de investigación, búsqueda y atención a las víctimas. Del caso Ayotzinapa a nuevos mecanismos en la lucha contra la impunidad. Temis.

Botero, J. \& Leal, Y. (2015). Sujeto y acción en el pensamiento político de Hannah Arendt. Signos filosóficos, 17(33), 52-76.

Bronfenbrenner, U. (1987). La ecología del desarrollo humano: experimentos en entornos naturales y deseados. Paidós.

Crozier, M. (1990). El actory el sistema. Las restricciones de la acción colectiva. Alianza Editorial.

Dorst, K., Kaldor, L., Klippan, L., Watson, R. (2016). Designing for the common good. BIS Publishers.

Ema, J. (2004). Del sujeto a la agencia (a través de lo político). Athene Digital, (6), 6-12.

Fajardo, L. (2009). A propósito de la comunicación verbal. Forma y Función, 22(2), 12-142.

García, E. (2007). El concepto de actor. Reflexiones y propuestas para la Ciencia Política. Andamios, 3(6), 199-216.

Giddens, A. (1984). The Constitution of Society. Outline of the Theory of Structuration. University of California Press.

Gutiérrez de Piñeras, B., Coronel, E. \& Pérez, C. (2009). Revisión teórica del concepto de victimización secundaria. Liberabit, 15(1), 49-58.

Hernández, I. (2019). La "atención estética": explorando las posibilidades del diseño de interacción para reducir la revictimización. Diseña, 15, 140-161.

Hernández, I. (2020). Pautas de institucionalización de la atención a víctimas en Ciudad Juárez [Tesis doctoral]. Universidad Autónoma de Ciudad Juárez. http://hdl. handle.net/20.500.11961/5854

Hernández, P. (2011). Centros de Justicia para las mujeres. Lineamientos urbanos y diseño arquitectónico. Programa de Naciones Unidas para los Asentamientos Humanos [ONU-Habitat]. México. https://www.gob.mx/cms/uploads/attachment/ file/63354/cjmlineamientos.pdf

Instituto Nacional de Estadística, Geografía e Informática [INEGI]. (2019). Encuesta Nacional de Victimización y Percepción sobre Seguridad Pública.
Principales Resultados. https://www.Inegi.org.mx/ contenidos/programas/Envipe/2019/doc/Envipe2019_ presentacion_nacional.pdf

Instituto Nacional de Estadística, Geografía e Informática [INEGI]. (2020). Encuesta Nacional de Victimización y Percepción sobre Seguridad Pública. Principales Resultados. https://www.inegi.org. $\mathrm{mx} /$ contenidos/programas/envipe/2020/doc/ envipe2020_presentacion_nacional.pdf

Lastra, M. R. (2011). El concepto de la conversación en la clase de comunicación oral. En M. Salas Díaz, M.J. Gómez del Castillo \& C. Morán Rodríguez (Eds.), XLVI Congreso. La cultura española, entre la tradición y la modernidad. Nuevos retos para la enseñanza del español (pp.161-172). Asociación Europea de Profesores de Español [AEPE]. https://cvc.cervantes. es/ensenanza/biblioteca_ele/aepe/pdf/congreso_46/ congreso_46_18.pdf

Leiva, E. (2015). Poder, agencia y empoderamiento. I Congreso Latinoamericano de Teoría Social. Instituto de Investigaciones Gino Germani. Facultad de Ciencias Sociales, Universidad de Buenos Aires, Buenos Aires. https://cdsa.aacademica.org/000-079/13

Manzini, E. (2015). Design, when everybody designs: An introduction to design for social innovation. MIT press.

Marchiori, H. (2007). Asistencia a víctimas. Respuestas desde la primera línea de atención. En H. Marchiori (Coord.), Principios de Justicia y Asistencia para las víctimas (pp.85-107). Encuentro.

Melup, I. (2007). Declaración de Naciones Unidas sobre Principios de Justicia para las víctimas del Delito y abuso de Poder. En H. Marchiori (Coord.), Principios de Justicia y Asistencia para las víctimas (pp.19-37). Encuentro.

Minoja, L. (2016). Lineamientos arquitectónicos para el diseño y construcción de refugios especializados para mujeres, sus hijas e hijos, víctimas de violencia extrema, familia y/o de género. Programa de las Naciones Unidas para los Asentamientos Humanos [ONU-Habitat]. https://www.gob.mx/cms/uploads/ attachment/file/226938/Lineamientos_arquitect_ nicos_Refugios_para_mujeres_sus_hijas_e_hijos_RED2017-1.pdf

Morillas, D. (2018). Victimización penitenciaria. Revista Internacional de Doctrina y Jurisprudencia, 14. DOI: http://dx.doi.org/10.25115/ridj.v5i14.1839

Molina, S. \& Sánchez, X. (2019). Hacia el diseño del siglo XX. ¿El diseñador como agente de cambio? En Ortiz Nicolás, J.C. \& Alatorre Guzmán, D. (Coords.), Innovación social y Diseño (pp.246-288. Universidad Nacional Autónoma de México. 
Open Society Foundations. (2016). Atrocidades innegables. Confrontando crímenes de lesa humanidad en México ( $2^{\mathrm{a}}$ ed.). https://www.justiceinitiative.org/ uploads/5d386d17-57aa-4b74-b896-43883af55574/ undeniable-atrocities-esp-2nd-edition.pdf

Ortiz, J.C. (2016). Diseñando el cambio. La innovación social y sus retos. Economía creativa, 6, 9-34.

Ortiz, J. C \& Hernández, I. (2019). La inserción de la disciplina del diseño en el reto de la victimización. Academia XXII, 10(19), 165-188. http://www.revistas. unam.mx/index.php/aca/article/view/69893

Ortiz, J. C., Hernández, I. \& Peinado, P. (2019). El modelo ecológico como herramienta para establecer el área de oportunidad en retos de diseño para la innovación social. En J.C. Ortiz Nicolás \& D. Alatorre (Coords.), Innovación social y Diseño (pp.90-124). Universidad Nacional Autónoma de México.

Real Academia Española. (2020). Diccionario de la lengua española. htttp://rae.es

Sánchez, D. A. F. (2018). Los derechos humanos de las víctimas del Delito: Garantismo victimal. Editorial Flores.

Sanz-Abbud, M. \& Saldarriaga, M. (2010). El diseño estratégico aplicado a la generación de soluciones innovadoras que incentiven la denuncia de los delitos en México. En B. Borja de Mozota (Ed.), Gestión del diseño. La forma de gestionar el uso del diseño para construir la marca de valor e innovación corporativa. (pp.360-365). Divine Egg Publicaciones. https://www. researchgate.net/publication/257578134

Thackara, J. (2013). Diseñando para un mundo complejo. Acciones para lograr la sustentabilidad. Designio.

Valenzuela, P. A. (2012). Mella, Marcelo (2012). Elementos de ciencia política: Conceptos, actores y procesos. Vol. 1. Santiago: Facultad de Humanidades, USACH, RIL Editores. Política. Revista de Ciencia Política, 50(2), 151-155.

Velasco, H. \& Díaz de Rada, A. (1997). La lógica de la investigación etnográca: un modelo de trabajo para etnógrafos de escuela. Trota.

World Design Organization [WDO]. (2020, agosto 3). UN Women and World Design Organization team up to develop innovative, human-centric design solutions to prevent violence against women and girls. https://wdo. org/un-women-and-world-design-organizationteam-up-to-develop-innovative-human-centricdesign-solutions-to-prevent-violence-againstwomen-and-girls/ 